\title{
Continuous-wave laser operation of a dipole antenna terahertz microresonator
}

\author{
Luca Masini ${ }^{1, \star}$, Alessandro Pitanti ${ }^{1, \star}$, Lorenzo Baldacci ${ }^{1}$, Miriam S Vitiello ${ }^{1}$, Riccardo Degl'Innocenti ${ }^{2}$, \\ Harvey E Beere ${ }^{2}$, David A Ritchie ${ }^{2}$ and Alessandro Tredicucci ${ }^{3}$
}

Resonators and the way they couple to external radiation rely on very different concepts if one considers devices belonging to the photonic and electronic worlds. The terahertz frequency range, however, provides intriguing possibilities for the development of hybrid technologies that merge ideas from both fields in novel functional designs. In this paper, we show that high-quality, subwavelength, whispering-gallery lasers can be combined to form a linear dipole antenna, which creates a very efficient, lowthreshold laser emission in a collimated beam pattern. For this purpose, we employ a terahertz quantum-cascade active region patterned into two $19-\mu \mathrm{m}$-radius microdisks coupled by a suspended metallic bridge, which simultaneously acts as an inductive antenna and produces the dipole symmetry of the lasing mode. Continuous-wave vertical emission is demonstrated at approximately $3.5 \mathrm{THz}$ in a very regular, low-divergence $\left( \pm 10^{\circ}\right)$ beam, with a high slope efficiency of at least $160 \mathrm{~mW} \mathrm{~A}^{-1}$ and a mere $6 \mathrm{~mA}$ of threshold current, which is ensured by the ultra-small resonator size $\left(\mathrm{V}_{\mathrm{RES}} / \lambda^{3} \approx 10^{-2}\right)$. The extremely low power consumption and the superior beam brightness make this concept very promising for the development of miniaturized and portable THz sources to be used in the field for imaging and sensing applications as well as for exploring novel optomechanical intracavity effects.

Light: Science \& Applications (2017) 6, e17054; doi:10.1038/lsa.2017.54; published online 6 October 2017

Keywords: microresonators; optical antennas; quantum cascade laser; terahertz; whispering-gallery

\section{INTRODUCTION}

Recent years have witnessed an increasing interest in terahertz $(\mathrm{THz})$ radiation technologies; research has ranged from the pure physics of electromagnetic waves between the optical and microwave regions to diverse applications in fields such as medical diagnostics, safety, quality control and observational astrophysics ${ }^{1,2}$. Remarkably, the particular wavelength of electromagnetic waves at these frequencies allows devices with hybrid configurations that are between photonics (wavelengths from hundreds of nanometers to several microns, which are typically smaller than the device) and electronics (wavelengths on the order of millimeters and longer, which are typically larger than the device), thereby interchanging and using concepts from both worlds ${ }^{3-5}$.

The coherent emission of THz-frequency light in cavities exploiting single-plasmon ${ }^{6}$ or micro-strip line architectures ${ }^{7}$ has been widely reported in the last decade. This is performed by exploiting quantum cascade semiconductor heterostructures, which rely on intersubband transitions between conduction energy levels ${ }^{8}$. The tight confinement of the resulting guided mode in the growth direction of the heterostructure ensures low lasing thresholds, but also results in poor out-coupling and detrimental large divergence of the emitted beam, which is mainly due to the diffraction of light at the device's facets (see, for example, Castellano et al. ${ }^{9}$ ).
One possible solution entails patterning the top metallic surface, to match the guided mode and the photons propagating outside, and simultaneously generating a collimated beam by interference ${ }^{10}$. In this line of thought, third-order distributed feedback (DFB) gratings ${ }^{11}$ in conventional ridge geometries have proven to be the ideal solution for ensuring efficient in-plane single-mode emission, even in very narrow strips with subwavelength lateral dimensions ${ }^{12}$. Alternatively, vertical lasers are obviously very appealing, but they present various challenges ${ }^{13}$ : Quantum Cascade Lasers (QCLs) only work in transversal magnetic (TM) polarization due to the intersubband selection rules, which means that vertical cavities are forbidden; ridge cavities are not desirable because they produce strongly elliptical divergence; and finally, in photonic structures that exploit second-order diffraction for extraction in the vertical direction, asymmetric modes are generally favored but display an emission minimum precisely $90^{\circ}$ from the surface due to far-field interference ${ }^{13}$. Photonic crystals, both periodic $^{14-16}$ and quasi-random ${ }^{17}$, have shown promising performance, but the beam profiles are still not ideal; they cannot be miniaturized and suffer from operation mostly under pulsed regimes. Diffraction-limited beam profiles can be obtained by employing external cavities based on metasurfaces ${ }^{18,19}$, but again, they cannot be monolithically integrated into subwavelength devices. Another promising approach is to engineer a set of short-cavity surface-

\footnotetext{
${ }^{1}$ NEST, CNR_-Istituto Nanoscienze and Scuola Normale Superiore, Piazza San Silvestro 12, 56127 Pisa, Italy; ${ }^{2}$ Cavendish Laboratory, University of Cambridge, JJ Thomson Avenue, Cambridge CB3 OHE, UK and ${ }^{3}$ NEST, CNR-Istituto Nanoscienze and Dipartimento di Fisica 'E. Fermi', Università degli studi di Pisa, 56127 Pisa, Italy

*These authors contributed equally to this work.

Correspondence: A Pitanti, Email: alessandro.pitanti@sns.it

Received 8 November 2016; revised 24 March 2017; accepted 26 March 2017; accepted article preview online 29 March 2017
} 
a

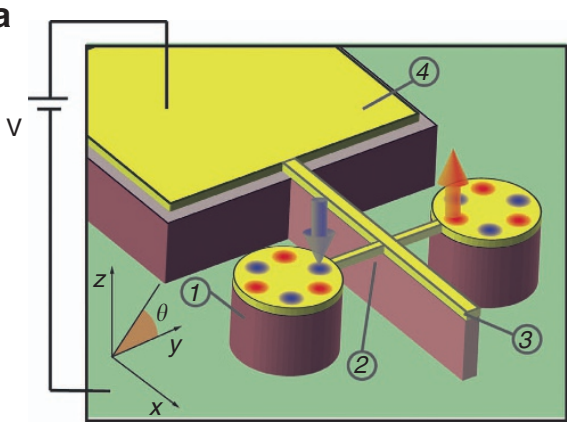

C

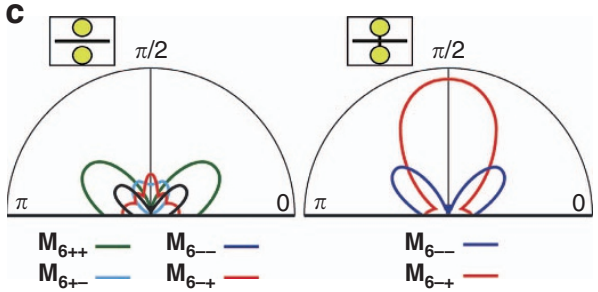

b

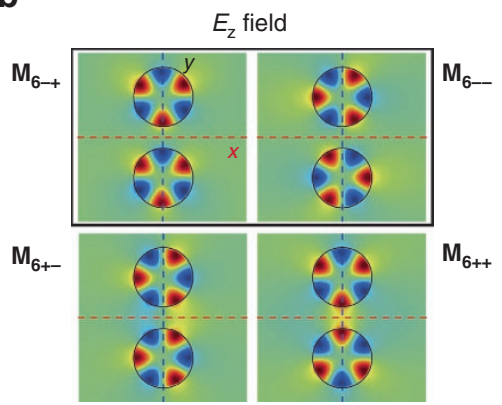

d

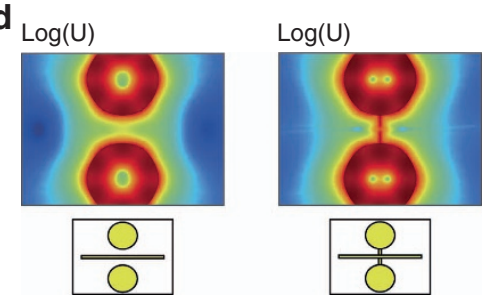

Figure 1 (a) Sketch of the device. Two etched WGM resonators (1) are connected by a suspended wire (2). A larger electrode (3) connects the resonant device to the bonding pad (4), where a bias can be applied from the top metal layer to the bottom ground plane. (b) Simulated $E_{z}$ for all the possible symmetry modes of the two coupled WGM resonators with no wire and with $n=6$. The odd modes for x-mirroring are the interesting ones in terms of vertical emission, and the ones naturally occurring in our device have a ground node in the center of the suspended wire. (c) Far-field emission (in the coordinate system of $\mathbf{a}$ for the simulated modes of $\mathbf{b}$ for devices without (left) and with (right) a suspended wire. (d) Energy profile of the $\mathbf{M}_{6-+}$ mode, on a log scale, for both cases. Note the large energy density along the connecting wire.

emitting distributed feedback QCLs with a definite phase relation that interact in the far field ${ }^{20}$. This concept has recently allowed highly collimated emission in the $\mathrm{THz}$ range under pulsed conditions.

Microdisk resonators operating on whispering-gallery modes (WGMs) are known to yield lasers with very low threshold currents thanks to their combination of a small size and high mode quality factors $^{21}$. For the same reason, however, efficient light extraction is a rather problematic issue and is even more so in the vertical direction ${ }^{22}$; a recent theoretical proposal suggested that the same metal-sandwiched microdisk cavity could act as a radiating patch antenna ${ }^{23}$, but the concept appears difficult to implement due to the high losses of the mode under consideration. Even more extreme miniaturization is possible by employing laser resonators based on inductance-capacitance (LC) circuits $^{5}$, but to date, this type of laser has displayed only very limited performance.

In this paper, we introduce a novel resonator concept that merges whispering-gallery cavities into a linear dipole antenna emitter. Our hybrid design combines the advantageous high quality factor of WGMs with the optimal feeding of their emissions into the regular far-field profile created by the deeply subwavelength antenna.

By placing two subwavelength microdisk resonators in close proximity, we induce the formation of supermodes, whose Q-factors (with simulated values of 39 and 41 for the lowest and the highest energy ones, respectively) easily guarantee lowthreshold continuous-wave lasing action near $3.5 \mathrm{THz}$. If the coupling between these resonators is 'guided' by a suspended metallic wire, dipole symmetry is imposed on the modes, which gives rise to a dipole antenna-like field pattern with a resulting strong out-of-plane emission. Additionally, our suspended wire naturally acts as an electrode that allows the QCL heterostructure to be biased without the need for potentially field-perturbing bond wires in contact with the disks.

\section{MATERIALS AND METHODS}

\section{Modeling and numerical simulations}

The device design was conceived from single double-metal microdisk resonators ${ }^{24,25}$. This geometry embeds the $11-\mu \mathrm{m}$-thick QCL active region within a cylindrical mesa structure, which is covered with a 200-nm-thick metal layer and placed over a metallic, semi-infinite ground plane. The resonator eigenfrequencies associated with the intersubband-allowed TM-polarized modes were computed with the open-system approximation using a finite element method solver, with assumed refractive indexes of 3.6 and $225-319 i$ for the semiconductor stack and the metal, respectively, at $3.5 \mathrm{THz}$. The simulations returned complex eigenvalues, from which the Q-factors were obtained by considering the ratio between the real part and twice the imaginary part of the eigenfrequencies. Because they were axially symmetric, all the eigenmodes belonged to the $\mathbf{C}_{\mathbf{n}}$ group, which is the group of rotations with angle $2 \pi / n$. In the case of a microdisk with a radius of $20 \mu \mathrm{m}$, the WGMs with frequencies near $3.5 \mathrm{THz}$ belong to the $\mathbf{C}_{\mathbf{3}}$ group, which means that the field components have six nodes and anti-nodes (three positive and three negative).

If two such resonators are then placed laterally close to each other, the resonant modes couple. The resultant supermodes can be even or odd with respect to mirroring along the $x$ - or the $y$-axis (in the coordinate system shown in Figure 1a), which results in four possible mode combinations (Figure $1 \mathrm{~b}$ ), which we label $\mathbf{M}_{2 n, \pm \pm}$, where $2 n$ indicates the number of anti-nodes of the mode of a single disk and the first (second) \pm indicates even or odd symmetry with respect to the $x$ - $(y$-)axis. Once the eigenmodes have been computed, their respective far fields can be extracted by employing the Stratton-Chu formula ${ }^{26}$. Their intensity profiles (Figure $1 \mathrm{c}$, left panel) show that most of the modes presented strong radial contributions and, in general, that the emission in the vertical direction was strongly suppressed. However, when the two microdisks were coupled through a suspended metallic wire (in our case, the wire was $14.5-\mu \mathrm{m}$-long, 

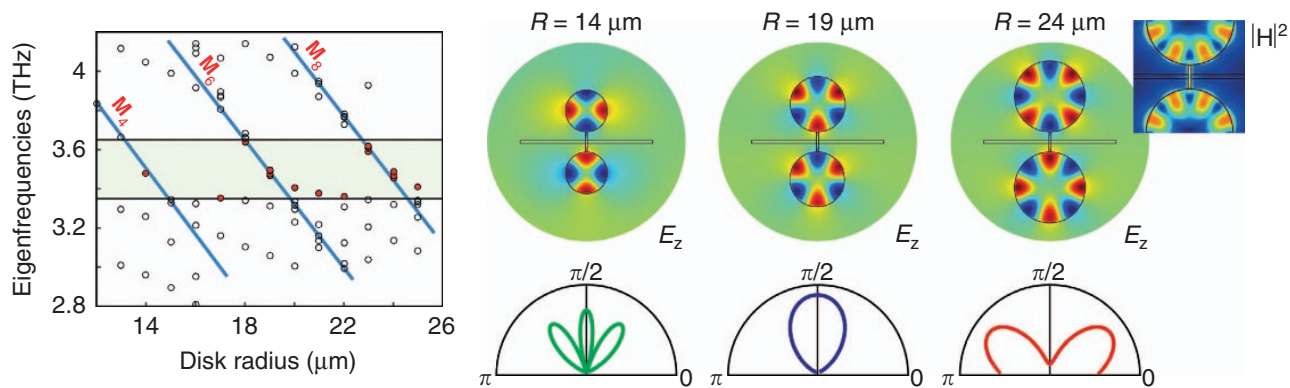

Figure 2 Dispersion of high-quality modes $(Q>35)$ as a function of the radius of the disks. We highlight the modes that fall closer to the spectral gain peak (shaded area) and are likely to give rise to lasing action with a red circle. The dispersion of modes belonging to different symmetry groups is almost linear (see the blue lines for the eye), but it is modified due to hybridization with modes localized on the long contact electrode. For selected modes of different radius (and radial families), electric field maps and far-field emissions are also reported for the wire geometry (14.5- $\mu \mathrm{m}$-long, $470-\mathrm{nm}$-wide, and 200-nmthick) shown in Figure 1. In the inset, an enlargement of the norm of the magnetic field around the wire is shown for the device with a radius of $24 \mu \mathrm{m}$.

470-nm-wide, and 200-nm-thick—see the device sketch in Figure 1a), the mode behavior changed drastically. The presence of the wire indeed introduced multiple features. The most important one was local enhancement of the resonator coupling with a consequent increase of the electromagnetic energy density between them. This can be best appreciated by comparing the energy density $U=\frac{\epsilon}{2}|\vec{E}|^{2}+\frac{\mu}{2}|\vec{H}|^{2}$ plots for mode $\mathbf{M}_{6-+}$ computed without (left panel of Figure 1d) and with the suspended wire (right panel of Figure 1d). Second, the wire bridge was able to directly bias both resonators by simply being connected to a bonding pad through a large, non-suspended electrode (Figure 1a); this configuration had the added benefit of creating a virtual ground precisely between the two resonators and then killing the $x$-even modes in the real device for symmetry reasons. As is clearly visible in the right panel of Figure 1c, adding the suspended wire strongly modified the far-field pattern of $\mathbf{M}_{\mathbf{6 - +}}$, which then showed a strong vertical emission with small divergence. This effect can be qualitatively understood by looking at the resonance field distribution, which matched that of a horizontal dipole with the corresponding radiation profile.

Interestingly, this peculiar behavior was activated only when the antenna coupling between the resonators and the radial losses was properly balanced. As expected in laser systems, the modes with sufficiently high Q-factors that were spectrally close to the gain peak were the most likely to lase. As can be qualitatively seen in Figure 2, by changing the radii of the microdisks, we were able to push modes belonging to different symmetry groups (different values of $n$ and different radial families) within the spectral region of large active gain (the shaded rectangle in the figure). Because it has a different modal shape, each radial family possesses its own coupling and radiative loss rate; these combine to determine the far-field pattern. In particular, we show the emission properties of selected devices with different disk diameters and the connecting wire geometry discussed above for Figure 1 in the right-hand side of Figure 2. At a fixed resonance frequency of approximately $3.5 \mathrm{THz}$, as imposed by the heterostructure's gain spectrum, the next lasing mode for decreasing radii was expected to belong to family $\mathbf{M}_{\mathbf{4}}$ (the leftmost panel in Figure 2). Smaller radii led to increased radiative losses in the WGMs, which then exceeded the strength of the mode coupling; as a consequence, the emission due to mode $\mathbf{M}_{4-+}$ included a strong radial component with a residual vertically out-coupled contribution due to the wireinduced dipole effect. Something similar also occurred in the opposite direction when the radius was too large (the rightmost panel in Figure 2), which obviously led to tighter mode confinement and less coupling. The far field for mode $\mathbf{M}_{\mathbf{8 -}}$ in microdisks with a radius of $24 \mu \mathrm{m}$ remained strongly radial with, again, only a residual field amplitude at $\theta=\pi / 2$. The optimal condition was found when the radius was approximately $19 \mu \mathrm{m}$ (as in our device), which allowed the bending loss to match the coupling strength (in a sort of critical coupling regime), giving rise to a single-lobe, strong vertical emission, as shown in the central panel of Figure 2.

\section{Device fabrication and testing}

As the active core, we selected an 11- $\mu$ m-thick GaAs/AlGaAs QCL heterostructure exploiting a bound-to-continuum design with a phonon extraction/injection stage ${ }^{27}$. More details of the sample and its gain performance can be found elsewhere ${ }^{28}$.

After the wafer was bonded to a highly doped host substrate using a $\mathrm{Cr} / \mathrm{Au}$ layer, the structure was mechanically lapped and wet-etched to remove the original substrate and provide access to the highly doped contact layer of the active region. A 400-nm-thick insulating layer of $\mathrm{SiO}_{2}$ was then sputtered using a 2-nm thermally evaporated $\mathrm{Cr}$ layer to improve adhesion. This step was crucial for insulating the bonding pad ( 4 in Figure 1a) and the connecting electrode ( 3 in Figure 1a) to confine the current flow within the microdisk area. Employing a negative resist (AllResist (GmbH, Strausberg, Germany) ma-N 2403), we then defined a polymeric mask shaped to match the bonding pad and connecting electrodes, and subsequently etched the insulating layer with a buffered-oxide etching (BOE) solution. The wet etching naturally smoothed the edges of the $\mathrm{SiO}_{2}$ enough to produce a continuous metal film in the subsequent deposition. The last step required further e-beam lithography (AllResist PMMA 679.04 mask) to define all the device components-microdisks, connecting wire, electrode and bonding pad-followed by thermal evaporation of the $\mathrm{Cr} / \mathrm{Au} / \mathrm{Ni}$ 10/200/20 nm metal layers. The Ni layer acted as further protection for the following etching, which was performed with an inductively coupled plasma reactive ion etching machine (ICP RIE from Sentech instruments, GmbH, Berlin, Germany) using a mixture of $\mathrm{BCl}_{3}, \mathrm{Cl}_{2}$ and $\mathrm{Ar}(8 / 10 / 6)$. The latter recipe was calibrated to provide a slight undercut; in this way, the thin connecting wire was automatically suspended between the electrode contact and the microdisks.

\section{RESULTS AND DISCUSSION}

The fabricated device (Figure 3a) was mounted in a continuous-flow liquid-He cryostat and driven either by continuous-wave (when the spectra were collected) or by directly modulating the bias current at $33 \mathrm{~Hz}$. The latter choice was made to maximize the responsivity of the 
b
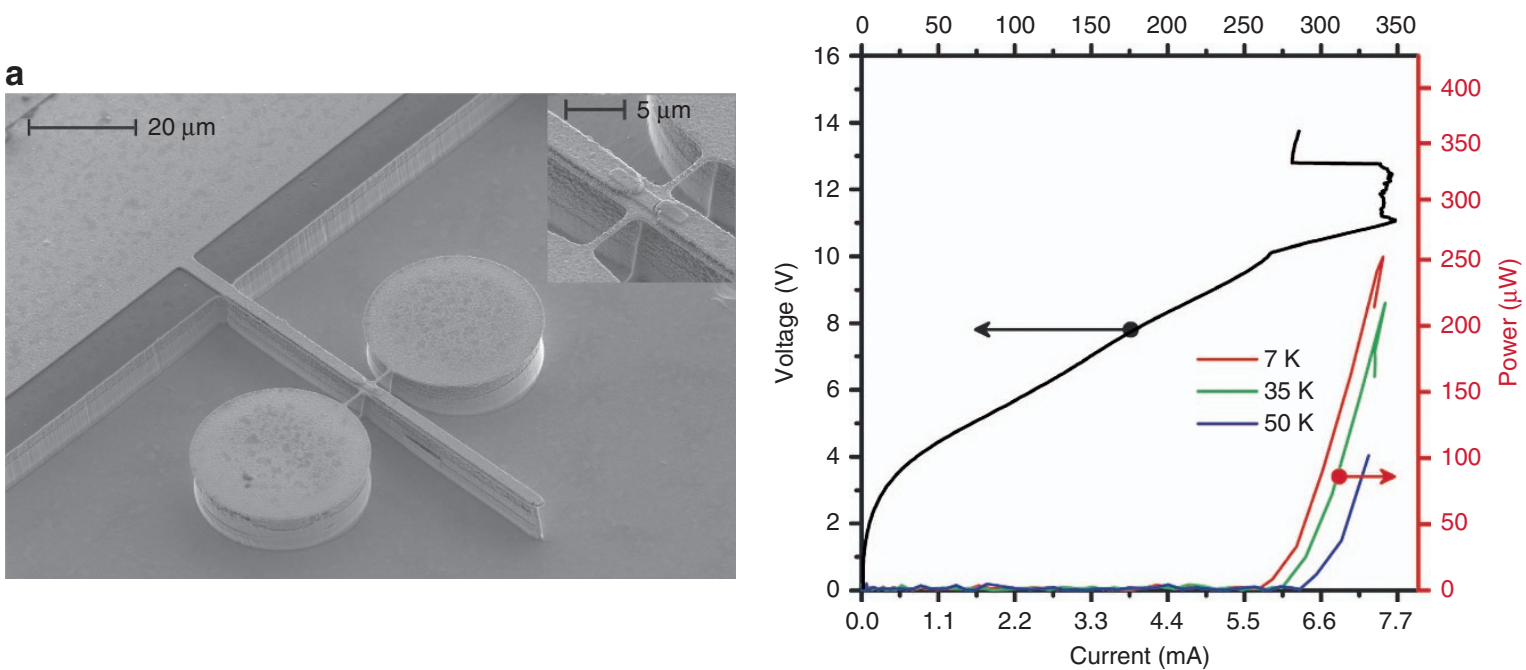

Figure 3 (a) Scanning electron microscope image of the microresonator. Inset: an enlargement of the suspended wire. (b) Current-voltage (IV) and lightcurrent (LI) characteristics. The IV was recorded with a DC current bias and is practically unchanged for every chosen temperature even when the bias is modulated at $33 \mathrm{~Hz}$. The LI curves are shown for three operating temperatures and are a modulated drive current.

pyroelectric detector (PYRO 1000 from Infrared System Development, Goldenrod, FL, USA) and use a lock-in detection technique (which was particularly necessary in the far-field measurements). Note that the detector was preliminarily tested against a properly companycalibrated $\mathrm{THz}$ pyroelectric detector (GENTEC-EO, INC., Lake Oswego, OR, USA). The voltage drop across the device was measured using a 4-wire connection scheme, and the total emitted power was obtained by integrating the far field scanned at a distance of $7 \mathrm{~cm}$ and a collection angle of $50^{\circ}$.

As shown in Figure 3b, a remarkably low lasing threshold was observed. It varied from 5.7 to $6.3 \mathrm{~mA}$ (which corresponded to a current density of $250-275 \mathrm{~A} \mathrm{~cm}^{-2}$ ) as a function of the heat sink temperature. Despite its very subwavelength size, with a resonator volume of $2 \times 11 \mu \mathrm{m} \quad\left(\pi R^{2}\right) \approx 2.5 \times 10^{4} \mu \mathrm{m}^{3}$ at a wavelength of $\lambda^{3} \approx 1 \times 10^{6} \mu \mathrm{m}^{3}$, the laser provided strong emission with an integrated power of up to $220 \mu \mathrm{W}$, a slope efficiency of approximately $160 \mathrm{~mW} \mathrm{~A}^{-1}$, and a wall-plug efficiency of approximately $0.26 \%$.

The measured threshold current at $7 \mathrm{~K}$ was slightly smaller than the one observed for a ridge waveguide fabricated with the same heterostructure as a reference $\left(300 \mathrm{~A} \mathrm{~cm}^{-2}\right.$ at $\left.10 \mathrm{~K}\right)$. Although this difference could be attributed to the larger Q-factor of our resonator, uncertainties spanning from the estimate of the active area and the actual laser operating temperature did not allow us to make any quantitative conclusion regarding comparing the thresholds of in the two devices.

The $10 \mathrm{~K}$ emission spectrum near maximum power ( $13 \mathrm{~V}$ bias, see Figure $4 \mathrm{a}$ ) shows two lasing modes, which can be attributed to mode $\mathbf{M}_{6--}$ (weaker peak) and to mode $\mathbf{M}_{6-+}$ (stronger peak); they are expected to be simultaneously active in the far field with relative contributions that are proportional to the ratio of the intensities of the related emission lines $I_{\mathbf{M}_{6--}} / I_{\mathbf{M}_{6-+}}=0.04$. Whereas mode $\mathbf{M}_{6--}$ had a slightly lower threshold than mode $\mathbf{M}_{6-+}$, when the injection bias was increasing, the $\mathbf{M}_{\mathbf{6 - +}}$ peak quickly dominated the spectrum, thanks to the better output and collection efficiency of the collimated beam (see the inset in Figure 4a). It is worth adding that the spectral measurements in Figure 4 were collected by an $f / 1$ parabolic mirror placed at the same distance from the pyroelectric detector as in the far- field experiment. The collection angle was then roughly the same as that scanned in the far-field measurements. Although it is true that this was a rough approximation, it was at least reasonable to assume that the relative contribution of the modes to the far-field measurements corresponded to the relative intensity recorded in the spectra.

The emission patterns were collected at a distance of $7 \mathrm{~cm}$, and IV and LI curves were simultaneously enacted to examine the complete evolution of the emission with the laser driving conditions. Whereas we saw a mostly diffused pattern just above the threshold, as soon as mode $\mathbf{M}_{6-+}$ became dominant, an intense central spot with a divergence of $\pm 10^{\circ}$ appeared in the vertical direction (Figure $4 \mathrm{~b}$ ). The simulated (Figure 4c) far-field maps were created by extracting the field components of modes $\mathbf{M}_{\mathbf{6 - -}}$ and $\mathbf{M}_{\mathbf{6 - +}}$ using the Stratton-Chu formula and weighting the respective intensity maps with the intensity ratio deduced from the spectral measurements (see above). The comparison with Figure $4 \mathrm{~b}$ shows nearly perfect agreement with the experimental emission profile.

\section{CONCLUSIONS}

Combining the optimal photonic properties of WGM resonators and the efficient radiation patterns provided by metallic antennas, we realized subwavelength $\mathrm{THz}$ microresonators that display extremely low laser thresholds $(<6 \mathrm{~mA})$ with $220 \mu \mathrm{W}$ vertical output power in continuous-wave and in a very regular beam profile. Together with the expected benefits for practical applications that require miniature $\mathrm{THz}$ sources featuring low power consumption and high brightness, the proposed device concept opens the path to more visionary perspectives by borrowing concepts that traditionally belong to different fields. Intriguingly, a device with a similar geometry could indeed be used for experiments in which mechanical vibrations affect the laser action itself $^{29}$. It has been seen that the fundamental optical mode in conceptually similar devices is somewhat related to the one of LC resonators ${ }^{5}$ in which the two regions of the heterostructure behave as capacitors connected by inductance, which, in our case, is the suspended wire. Note that for the geometrical parameters reported in this manuscript, the simulated frequency of the LC-like mode is at 
a

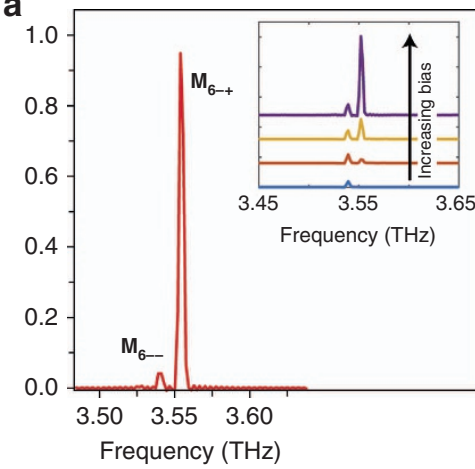

b

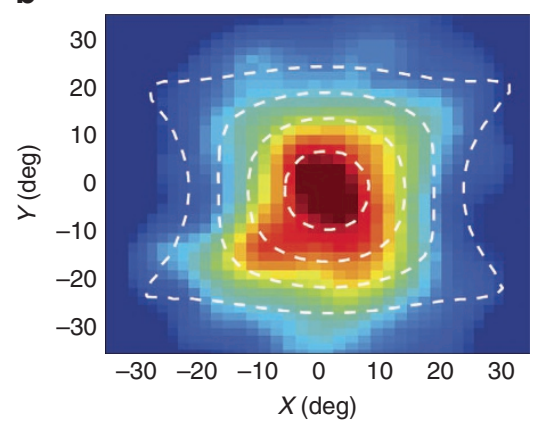

C

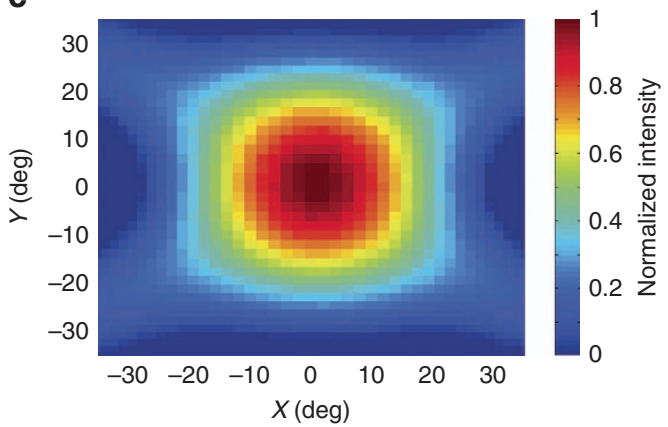

Figure 4 (a) Laser emission spectrum collected using a Fourier-transform infrared spectrometer in rapid scan mode with a DTGS internal detector and a parabolic gold mirror with a focal length of $50 \mathrm{~mm}$ while the QCL was driven at $13 \mathrm{~V}$ at a heat sink temperature of $10 \mathrm{~K}$. Inset: evolution of the spectral emission as the bias is increased from slightly above the threshold (10.8 V) to $11 \mathrm{~V}$. From 11 to $13 \mathrm{~V}$, the spectral shape is the same. (b) Measured far-field pattern collected with a pyroelectric detector mounted on a motorized stage control and placed at a distance of $7 \mathrm{~cm}$ from the laser surface plane under the same experimental conditions as in a. To completely evaluate the whole emission pattern, a scan range of $11 \mathrm{~cm}$ was chosen. The origin of the coordinate system corresponds to the vertical direction. Five-point smoothing was introduced to account for the large sensitive area of the detector used. The white isolines were extracted from the simulations reported in (c). We incoherently added the far-fields relating the $\mathbf{M}_{6-.}$ and $\mathbf{M}_{6-+}$ modes by considering their relative intensities, which were obtained from the emission spectrum.

approximately $0.38 \mathrm{THz}$, which is too far from the spectral gain peak to expect any lasing action; an appropriate rescaling of the geometric size is required to push the mode to higher frequencies.

If lasing action in the LC mode were indeed achieved in our geometry, one would be able to achieve a system in which the inductance changes with the distance between the wire and the ground plane and overlaps with nearly half of the mode's total energy. Its vibrations could then lead to interesting phenomena, such as stabilization of the lasing mode and frequency tuning.

\section{CONFLICT OF INTEREST}

The authors declare no conflict of interest.

\section{ACKNOWLEDGEMENTS}

The authors acknowledge funding from EU through the ERC Advanced Grant SouLMan (GA 321122).

1 Siegel PH. Terahertz technology. IEEE Trans Microw Theory Tech 2002; 50: 910-928.

2 Tonouchi M. Cutting-edge terahertz technology. Nat Photonics 2007; 1: 97-105.

3 Lewis RA. A review of terahertz sources. J Phys D 2014; 47: 374001.

4 Strupiechonski E, Xu G, Cavalié P, Isac N, Dhillon S et al. Hybrid electronic-photonic subwavelength cavities operating at terahertz frequencies. Phys Rev B 2013; 87: 041408.

5 Walther C, Scalari G, Amanti MI, Beck M, Faist J. Microcavity laser oscillating in a circuit-based resonator. Science 2010; 327: 1495-1497.

6 Köhler R, Tredicucci A, Beltram F, Beere HE, Linfield EH et al. Terahertz semiconductorheterostructure laser. Nature 2002; 417: 156-159.

7 Williams BS, Kumar S, Callebaut H, Hu Q, Reno JL. Terahertz quantum-cascade laser at $\lambda \approx 100 \mu \mathrm{m}$ using metal waveguide for mode confinement. Appl Phys Lett 2003; 83. 2124-2126.

8 Faist J. Quantum Cascade Lasers. Oxford: Oxford University Press; 2013.

9 Castellano F, Li LH, Linfield EH, Davies AG, Beere HE et al. THz waveguide adapters for efficient radiation out-coupling from double metal THz QCLs. Opt Express 2015; 23: 5190-5200.

10 Vitiello MS, Tredicucci A. Tunable emission in THz quantum cascade lasers. IEEE Trans Terahertz Sci Technol 2011; 1: 76-84.

11 Amanti MI, Fischer M, Scalari G, Beck M, Faist J. Low-divergence single-mode terahertz quantum cascade laser. Nat Photonics 2009; 3: 586-590.

12 Amanti MI, Scalari G, Castellano F, Beck M, Faist J. Low divergence terahertz photonicwire laser. Opt Express 2010; 18: 6390-6395.

13 Mahler L, Tredicucci A. Photonic engineering of surface-emitting terahertz quantum cascade lasers. Laser Photonics Rev 2011; 5: 647-658.
14 Chassagneux Y, Colombelli R, Maineult W, Barbieri S, Beere HE et al. Electrically pumped photonic-crystal terahertz lasers controlled by boundary conditions. Nature 2009; 457: 174-178.

15 Chassagneux Y, Colombelli R, Maineult W, Barbieri S, Khanna SP et al. Graded photonic crystal terahertz quantum cascade lasers. Appl Phys Lett 2010; 96: 031104.

16 Diao ZL, Bonzon C, Scalari G, Beck M, Faist J et al. Continuous-wave vertically emitting photonic crystal terahertz laser. Laser Photonics Rev 2013; 7: L45-L50.

17 Vitiello MS, Nobile M, Ronzani A, Tredicucci A, Castellano F et al. Photonic quasicrystal terahertz lasers. Nat Commun 2014; 5: 5884.

18 Xu LY, Curwen CA, Hon PWC, Chen QS, Itoh T et al. Metasurface external cavity laser. Appl Phys Lett 2015; 107: 221105.

$19 \mathrm{Xu} \mathrm{L}$, Chen D, loth T, Reno JL, Williams BS. Focusing metasurface quantumcascade laser with a near diffraction-limited beam. Opt Express 2016; 24: 24117-24128.

20 Kao TY, Reno JL, Hu Q. Phase-locked laser arrays through global antenna mutual coupling. Nat Photonics 2016; 10: 541-546.

21 Chassagneux Y, Palomo J, Colombelli R, Dhillon S, Sirtori C et al. Terahertz microcavity lasers with subwavelength mode volumes and thresholds in the milliampere range. Appl Phys Lett 2007; 90: 091113.

22 Mahler L, Tredicucci A, Beltram F, Walther C, Faist J et al. Vertically emitting microdisk lasers. Nat Photonics 2009; 3: 46-49.

23 Andronov AA, Dodin EP, Nozdrin YN, Pozdnyakova VI, Sadofiev YG et al. Modes, emission beams and losses of $\mathrm{THz}$ heterostructure disk lasers: single-mode laser with vertical beam option. Electron Lett 2016; 52: 383-385.

24 Fasching G, Benz A, Unterrainer K, Zobl R, Andrews AM et al. Terahertz microcavity quantum-cascade lasers. Appl Phys Lett 2005; 87: 211112.

25 Fasching G, Deutsch C, Benz A, Andrews AM, Klang P et al. Electrically controllable photonic molecule laser. Opt Express 2009; 17: 20321-20326.

26 Stratton JA, Chu LJ. Diffraction theory of electromagnetic waves. Phys Rev 1939; 56 : 99-107.

27 Amanti MI, Scalari G, Terazzi R, Fischer M, Beck M et al. Bound-to-continuum terahertz quantum cascade laser with a single-quantum-well phonon extraction/injection stage. New J Phys 2009; 11: 125022.

28 Degl'Innocenti R, Shah YD, Masini L, Ronzani A, Pitanti A et al. Hyperuniform disordered terahertz quantum cascade laser. Sci Rep 2016; 6: 19325.

29 Baldacci L, Pitanti A, Masini L, Arcangeli A, Colangelo F et al. Thermal noise and optomechanical features in the emission of a membrane-coupled compound cavity laser diode. Sci Rep 2016; 6: 31489.

(i) (2) This work is licensed under a Creative Commons AttributionBY NC SA NonCommercial-ShareAlike 4.0 International License. The images or other third party material in this article are included in the article's Creative Commons license, unless indicated otherwise in the credit line; if the material is not included under the Creative Commons license, users will need to obtain permission from the license holder to reproduce the material. To view a copy of this license, visit http:// creativecommons.org/licenses/by-nc-sa/4.0/

(C) The Author(s) 2017 